\title{
Mechanically tunable optofluidic distributed feedback dye laser
}

\author{
Zhenyu Li, Zhaoyu Zhang, Axel Scherer, and Demetri Psaltis \\ Department of Electrical Engineering, California Institute of Technology, Pasadena, California 91125 \\ zhenyu@caltech.edu
}

\begin{abstract}
A continuously tunable optofluidic distributed feedback (DFB) dye laser was demonstrated on a monolithic replica molded poly(dimethylsiloxane) (PDMS) chip. The optical feedback was provided by a phase-shifted higher order Bragg grating embedded in the liquid core of a single mode buried channel waveguide. Due to the soft elastomeric nature of PDMS, the laser frequency could be tuned by mechanically stretching the grating period. In principle, the mechanical tuning range is only limited by the gain bandwidth. A tuning range of nearly $60 \mathrm{~nm}$ was demonstrated from a single dye laser chip by combining two common dye molecules Rhodamine 6G and Rhodamine 101. Single-mode operation was maintained with less than $0.1 \mathrm{~nm}$ linewidth. Because of the higher order grating, a single laser, when operated with different dye solutions, can provide tunable light output covering the entire spectrum from near UV to near IR in which efficient laser dyes are available. An array of five DFB dye lasers with different grating periods was also demonstrated on a chip. Such tunable integrated laser arrays are expected to become key components in inexpensive advanced spectroscopy chips.
\end{abstract}

(C)2006 Optical Society of America

OCIS codes: (140.2050) Dye lasers; (140.3600) Lasers, tunable; (140.3490) Lasers, distributedfeedback; (140.3570) Lasers, single-mode; (230.7380) Waveguides, channeled; (130.3120)

Integrated optics devices

\section{References and links}

1. B. Helbo, A. Kristensen, and A. Menon, "A micro-cavity fluidic dye laser," J. Micromech. Microeng. 13, 307-311 (2003).

2. D.V. Vezenov, B.T. Mayers, R.S. Conroy, G.M. Witesides, P.T. Snee, Y. Chan, D.G. Nocera, and M.G. Bawendi, "A low-threshold, high-efficiency microfluidic waveguide laser," J. Am. Chem. Soc. 127(25), 8952-8953 (2005).

3. J.C. Galas, J. Torres, M. Belotti, Q. Kou, and Y. Chen, "Microfluidic tunable dye laser with integrated mixer and ring resonator," App. Phys. Lett. 86, 264101 (2005).

4. B. Bilenberg, B. Helbo, J.P. Kutter and A. Kristensen, "Tunable Microfluidic Dye Laser," Proceedings of the 12th Int. Conf. on Solid-State Sensors, Actuators and Microsystems, Transducers, 206-209 (2003).

5. D. Psaltis, S.R. Quake and C. Yang,, "Developing optofluidic technology through the fusion of microfluidics and optics," Nature 442, 381-386, (2006).

6. Z. Li, Z. Zhang, T. Emery, A. Scherer and D. Psaltis, "Single mode optofluidic distributed feedback dye laser," Opt. Express 14, 696-701 (2006).

7. A. Yariv, Optical Electronics in Modern Communications (Oxford, New York, 1997).

8. M.A. Unger, H.P. Chou, T. Thorsen, A. Scherer, S.R. Quake, "Monolithic microfabricated valves and pumps by multilayer soft lithography," Science 288, 113-116 (2000).

9. L.A. Wellerbrophy and D.G. Hall, "Analysis of wave-guide gratings - application of Rouard's method," J. Opt. Soc. Am. A 2, 863-871 (1985).

10. C.V. Shank, J.E. Bjorkholm and H. Kogelnik, "Tunable distributed-feedback dye laser,” App. Phys. Lett. 18, 395-396 (1971).

11. J.C. McDonald and G.M. Whitesides, "Poly(dimethylsiloxane) as a material for fabricating microfluidic devices," Acc. Chem. Res. 35, 491-499 (2002).

12. W.T. Silfvast, Laser Fundamentals (Cambridge, Cambridge, 2004).

13. Y. Oki, S. Miyamoto, M. Maeda and N. J. Vasa, "Multiwavelength distributed-feedback dye laser array and its application to spectroscopy," Opt. Lett. 27, 1220-1222 (2002). 


\section{Introduction}

On-chip liquid dye lasers represent promising coherent light sources for 'lab-on-a-chip' systems in that they allow the integration of laser sources with other microfluidic and optical devices. Several groups have so far demonstrated such dye lasers by using different materials and laser cavity designs $[1,2,3]$. Tunable output can also be obtained using concentration or index tuning methods $[3,4]$. Indeed, on-chip liquid dye lasers are examples of the new class of emerging optofluidic devices, in which the integration of microfluidics with the adaptive nature of liquids enables unique performance that is not obtainable within solid state materials [5]. Recently, an optofluidic distributed feedback (DFB) dye laser was demonstrated on a monolithic poly(dimethylsiloxane) (PDMS) elastomer chip [6]. Stable single-mode operation with narrow linewidth was obtained using a phase-shifted higher order Bragg gating embedded in a single mode microfluidic channel waveguide. The use of PDMS and soft lithography results in inexpensive and easy fabrication, rapid device prototyping, biocompatibility and the opportunity to create disposable devices. In this paper, we demonstrate a tunable single-mode DFB dye laser that combines the mechanical flexibility of elastomer materials with the reconfigurability of the liquid dye gain medium.

\section{Chip design}

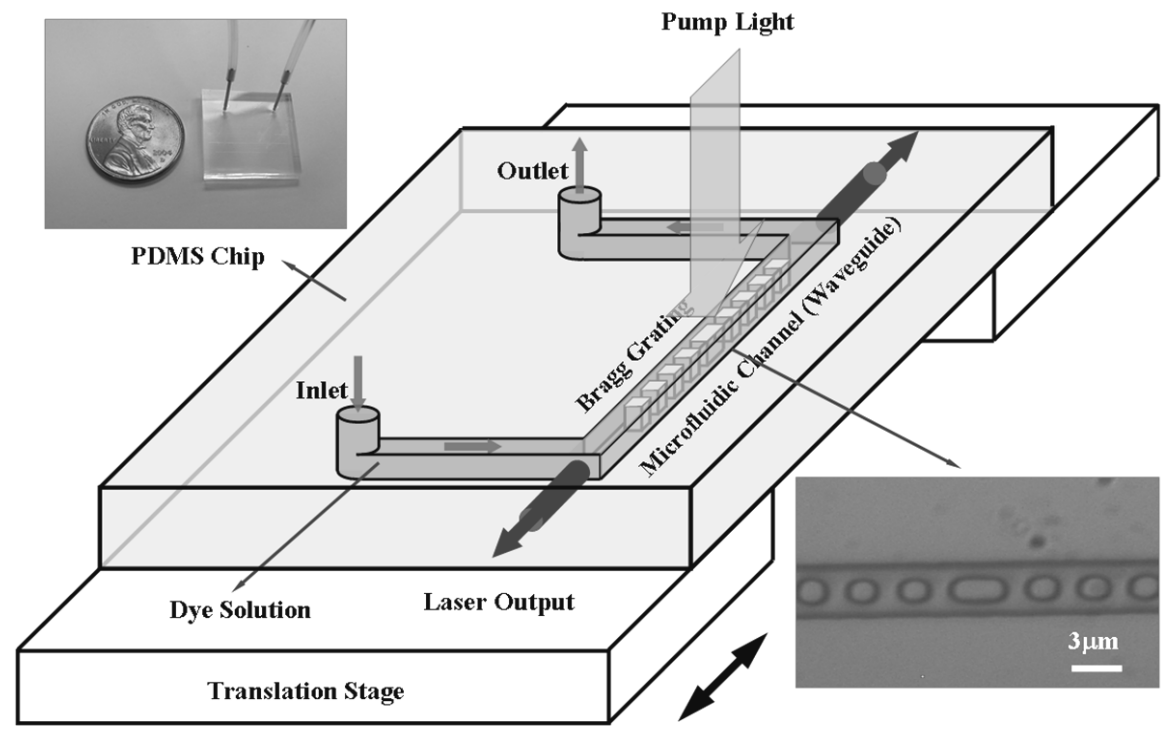

Fig. 1. Schematic diagram of a mechanically tunable optofluidic DFB dye laser chip. The upper inset shows an actual monolithic PDMS laser chip. The lower inset is an optical micrograph of the central phase-shifted region of the laser cavity. A Bragg grating with $3080 \mathrm{~nm}$ period is embedded in a $3 \mu \mathrm{m}$ wide microfluidic channel. The channel height is $2 \mu \mathrm{m}$. The size of the PDMS posts is about $1.28 \mu \mathrm{m} \times 1.8 \mu \mathrm{m}$ inferred from the optical micrograph. The central larger PDMS post introduces an effective $\pi / 2$ phase shift to ensure single wavelength lasing. The movement of the translation stage deforms the chip which causes the grating period to change.

As shown in Fig. 1, an optofluidic DFB dye laser was fabricated on a monolithic PDMS chip by replica molding, an inexpensive soft lithography technique described in Ref. [6]. When the microfluidic channel is subsequently filled with liquid of higher refractive index than that of PDMS (1.406, GE RTV615), a buried channel waveguide can be formed. The channel dimensions are $2 \mu \mathrm{m} \times 3 \mu \mathrm{m}$ and the index contrast is less than 0.003 so that the waveguide supports only the fundamental $\mathrm{TE}_{00}$ and $\mathrm{TM}_{00}$ modes. Distributed optical feedback is provided by defining periodic PDMS posts inside the channel with a $3080 \mathrm{~nm}$ period. This forms a $1 \mathrm{~cm}$ long 15th-order Bragg grating at wavelength approximately 570nm. The PDMS posts also provide mechanical support, preventing the microfluidic channel from collapsing. An 
effective $\pi / 2$ phase shift is introduced at the center of the grating to ensure single frequency operation at the Bragg wavelength [7]. The gain medium, a $2 \mathrm{mM}$ solution of Rhodamine 6G (Rh6G) or Rhodamine 101 (Rh101) in a methanol and ethylene glycol mixture with refractive index of 1.409, is then introduced into the flow channel to form the waveguide. To pump the dye molecules, 6ns Q-switched Nd:YAG laser pulses with $532 \mathrm{~nm}$ wavelength are focused through a cylindrical lens onto a $\sim 100 \mu \mathrm{m} \times 1 \mathrm{~cm}$ stripe aligned with the microfluidic channel. The fabrication, dye chemistry, and operation of the laser chip are fully compatible with silicone elastomer based microfluidics technology [8].

When a $\pi / 2$ phase-shifted DFB structure is used to provide the optical feedback, the lasing wavelength is determined by the Bragg condition:

$$
m \lambda_{m}=2 n_{\text {eff }} \Lambda
$$

where $\lambda_{m}$ is the $m$ th order resonant wavelength, $n_{e f f}$ is the effective index of the guided mode and $\Lambda$ is the grating period. Given $\Lambda=3080 \mathrm{~nm}$ and $n_{\text {eff }}=1.407$, the 15 th resonant wavelength and the free spectral range $(F S R)$ are $577.8 \mathrm{~nm}$ and $41.3 \mathrm{~nm}$ respectively. This large FSR ensures that at most two resonances can be simultaneously supported within the gain spectrum (typically $30-50 \mathrm{~nm}$ wide for dye molecules). Thus single frequency operation is obtained even at high pump levels due to gain discrimination. Figure 2 shows the simulated reflectivity spectrum of the overall structure using the Rouard's method [9]. The parameters used are: $\Lambda=$ $1280 \mathrm{~nm}+1800 \mathrm{~nm}$, grating length $\mathrm{L}=1 \mathrm{~cm}$, effective $\pi / 2$ phase shift at the center, core index $n_{\text {core }}=1.409$, and cladding/post index $n_{\text {clad }}=1.406$. Also shown in Fig. 2 are the normalized measured fluorescence spectra of Rh6G and Rh101 solutions used in the lasing experiment.

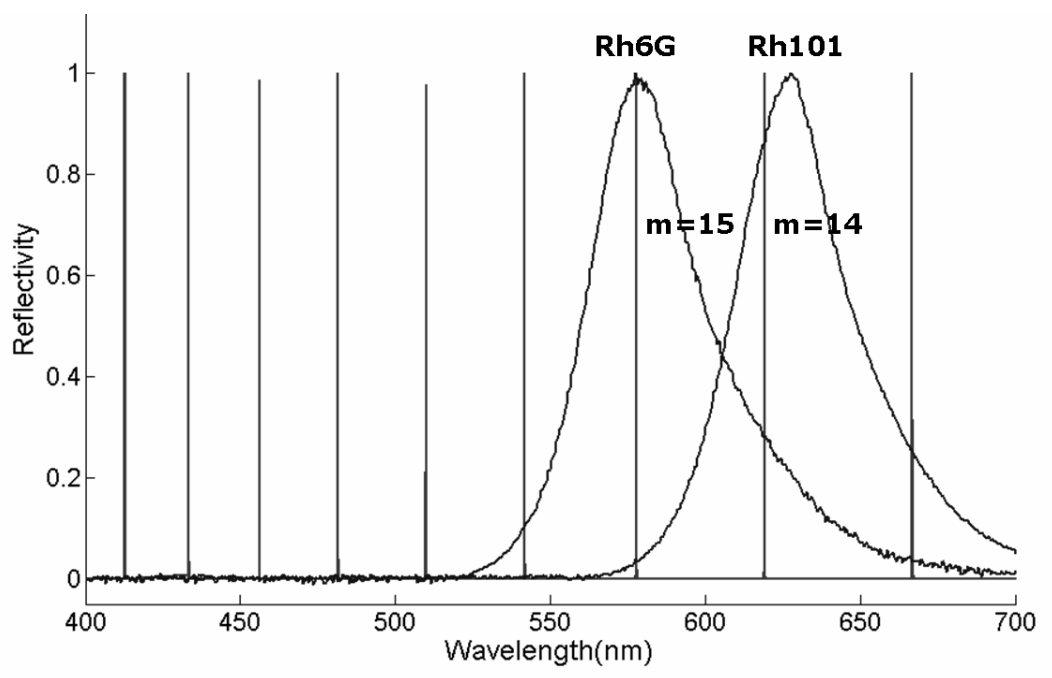

Fig. 2. Simulated reflectivity spectrum of a $\pi / 2$ phase shifted higher order DFB structure. The parameters used are given in the main text. Also shown are the normalized measured fluorescence spectra of Rh6G and Rh101 solutions used in the lasing experiment.

\section{Wavelength tuning}

The lasing wavelength can be tuned by changing either $n_{\text {eff }}, \Lambda$ or $m$ as has been demonstrated in conventional DFB dye lasers [10]. The effective index $n_{\text {eff }}$ can be varied by changing the core index or the cross sectional dimensions of the waveguide. However, the low Young's modulus of PDMS $(\sim 750 \mathrm{kPa})$ [11] enables a much more straight forward tuning method consisting of changing the grating period by simply stretching or compressing the elastomeric chip along the waveguide direction. Moreover, the grating order $m$ can be chosen by using different dye molecules whose emission spectra cover different spectral regions. The last two 
methods were used in this work to achieve a nearly $60 \mathrm{~nm}$ tuning range from yellow to red. As can be observed from Fig. 2, the potential tuning range for Rh6G and Rh101 is larger than $100 \mathrm{~nm}$ covering the spectrum from $550 \mathrm{~nm}$ to beyond $650 \mathrm{~nm}$. Actually, because of the multiple spectral resonances supported by the higher order grating, this laser cavity design can provide tunable output covering the entire available dye laser spectrum from $320 \mathrm{~nm}$ to 1200nm [12] when suitable dye molecules and pump light are selected. With a mixture of several dye molecules, simultaneous multiple color lasing from the same cavity is also possible.

\section{Results and discussion}
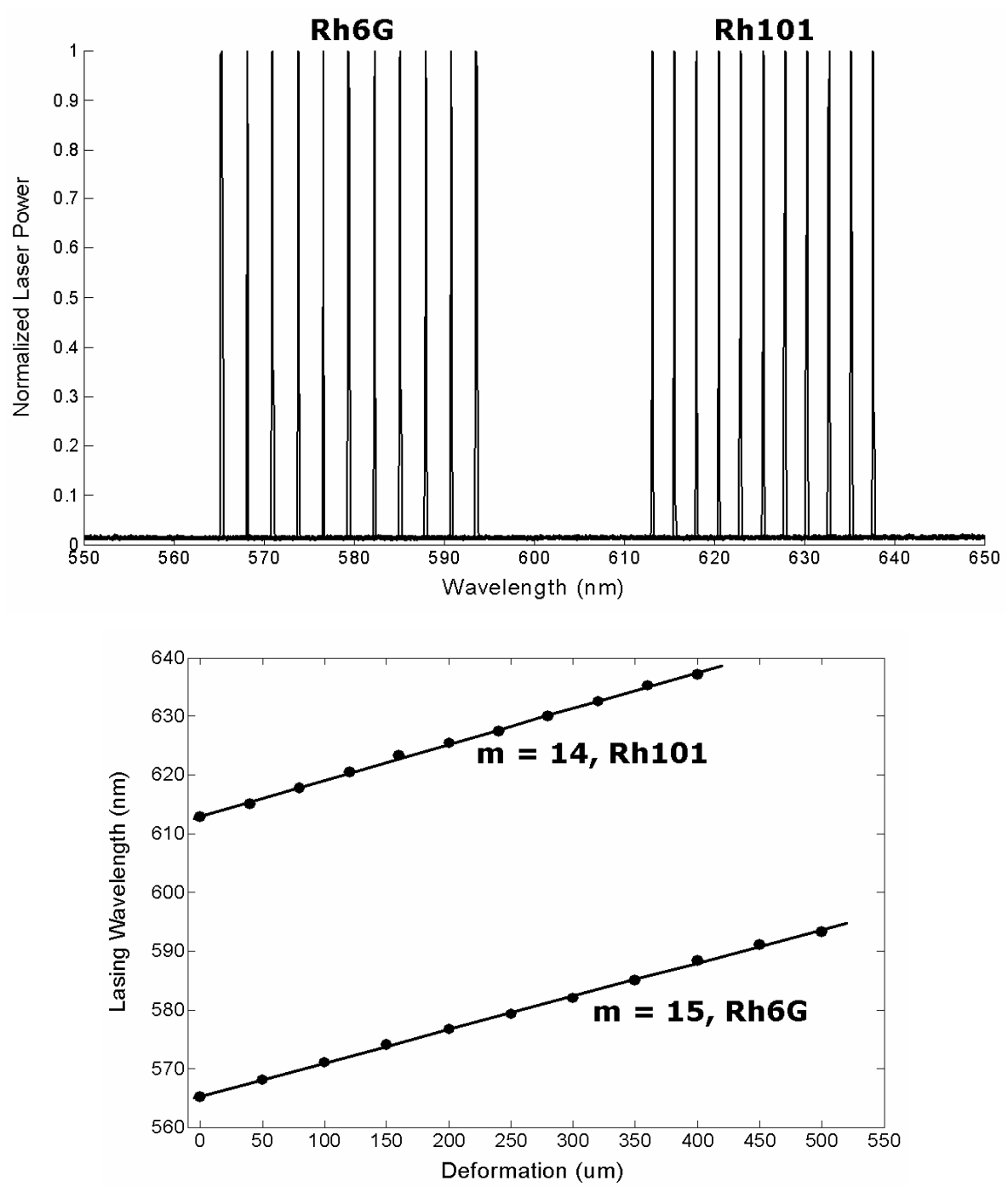

Fig. 3. Upper: normalized laser output of the mechanically tunable optofluidic DFB dye laser. Different peaks correspond to different grating periods. The measured laser linewidth is less than $0.1 \mathrm{~nm}$ throughout the tuning range. Lower: lasing wavelength versus the measured chip deformation. The points are the experimental data and the curve is the linear fit. The achieved single-mode tuning range for Rh6G is from $565 \mathrm{~nm}$ to $594 \mathrm{~nm}$ and is from $613 \mathrm{~nm}$ to $638 \mathrm{~nm}$ for Rh101.

To achieve mechanical tuning, the laser chip was glued to two micrometer stages with the laser region suspended in the center as shown in Fig. 1. One of the stages is a high resolution 
micrometer with $1 \mu \mathrm{m}$ sensitivity which provides accurate control and quantitative measurement of the deformation of the elastomer chip. The mechanical stages allow us to both stretch and compress the chip along the channel direction, and the result of mechanical cavity tuning is summarized in Fig. 3. The points on this Figure represent experimental data whereas the curves follow the linear fit. From this plot, the single mode tuning range for Rh6G covers a range from $565 \mathrm{~nm}$ to $594 \mathrm{~nm}$ whereas for Rh101, the tuning range extends from $613 \mathrm{~nm}$ to $638 \mathrm{~nm}$. A linear relationship between the lasing wavelength and the chip deformation was also observed in Fig. 3, indicating completely reversible elastic extension of the cavity. When the length of the central suspended region is $1 \mathrm{~cm}$, the total chip deformation required to traverse the above tuning ranges are about $500 \mu \mathrm{m}$ for $\mathrm{Rh} 6 \mathrm{G}$ and $400 \mu \mathrm{m}$ for Rh101. These macroscopic deformations correspond to $28 \mathrm{~nm}$ and $25 \mathrm{~nm}$ distortions of the grating period, respectively. Because of the extremely large available elastic deformation of PDMS of over $120 \%$, the ultimate tuning range is limited by the gain bandwidth of the laser dyes chosen. Only $\sim 5 \%$ deformation was used to achieve the $\sim 60 \mathrm{~nm}$ tuning range demonstrated in this experiment, and we believe that an even wider tuning range from $550 \mathrm{~nm}$ to $650 \mathrm{~nm}$ is obtainable with improved cavity design and a more uniform mechanical loading. The tuning is continuous and completely reversible, and no noticeable degradation of the chip was observed during a 5-cycle full range tuning test. Throughout the tuning range, stable single-mode operation was maintained with measured linewidth below $0.1 \mathrm{~nm}$, the resolution limited by our spectrometer (Ocean Optics HR4000).

The absorbed pump thresholds of this tunable dye laser are $\sim 150 \mathrm{~nJ}$ and $\sim 200 \mathrm{~nJ}$ for Rh6G and Rh101 respectively. As expected, we observe a decrease in the laser output power as the lasing wavelength is moved away from the peak in the gain spectrum in either direction. The deformation along the channel also causes the transverse waveguide dimensions to change slightly, resulting in changes the effective index of the guided mode. However, given that the Poisson's ratio of PDMS is approximately 0.5 , the estimated effective index change is only about $1.5 \times 10^{-5}$, the effect of lateral waveguide distortion on the lasing wavelength is negligible.
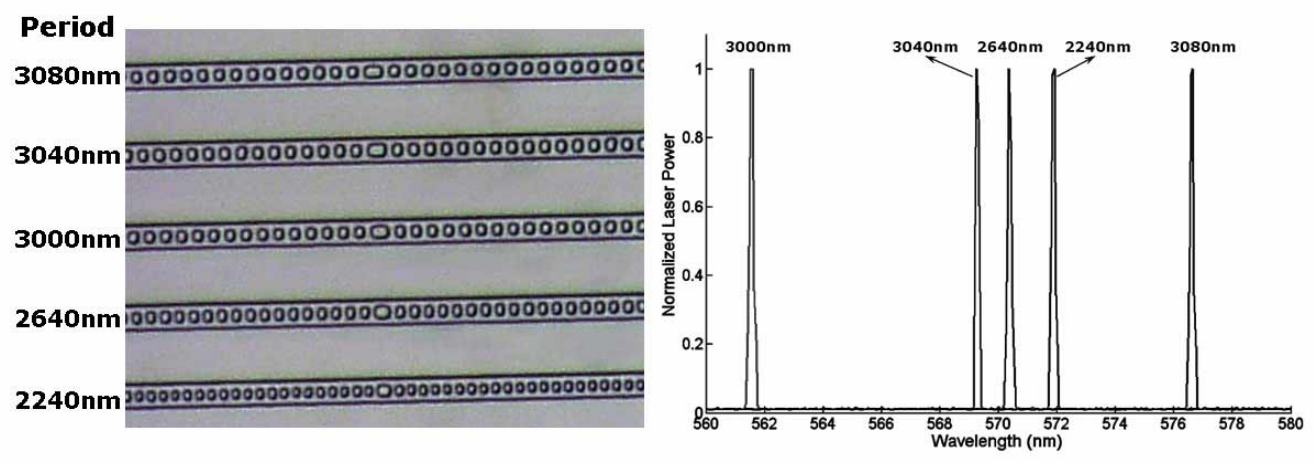

Fig. 4. Left: optical micrograph of an integrated array of five optofluidic DFB dye lasers. The grating period of each laser is given on the left. Right: normalized laser output of the array using Rh6G dye solution as the gain medium.

We also fabricated an array of five DFB dye lasers on a single PDMS chip. Figure 4 shows measured lasing results from this DFB array after filling the lasers with the Rh6G dye solution. Lasers with output wavelengths spanning a $\sim 15 \mathrm{~nm}$ range were measured from the DFB stripes with different grating periods. The low pump threshold $(<1 \mathrm{uJ})$ of each optofluidic DFB dye laser makes it possible to use a single high energy pulsed laser to pump hundreds of such lasers on a chip. This opens up the possibility of building highly parallel multiplexed biosensors on a chip ranging from applications as multiple-color flow cytometers and surface plasmon resonance based sensors to Raman spectroscopy sources and compact excitation spectroscopy systems. The introduction of replica molded multi-spectral sources in PDMS fluidic systems also provides an alternative to tunable lasers for constructing compact 
and inexpensive multi-wavelength scanning-less spectrometers integrated in microfluidic devices [13]. The low pump threshold enables the use of visible semiconductor laser diodes or even light emitting diodes as the pump sources to construct low-cost and compact portable spectrometers.

\section{Conclusions}

We have demonstrated a continuously tunable optofluidic DFB dye laser on a monolithic PDMS chip that uses simple mechanical deformation for accurate wavelength control. Singlemode operation was maintained throughout a $\sim 60 \mathrm{~nm}$ tuning range. Due to the higher order of the DFB grating in our devices, a single laser is capable of generating tunable output covering the spectral region from near ultra-violet to near infrared when a UV pump source is used. An integrated multi-spectral DFB laser array was also demonstrated by replica molding. Such laser arrays can be used to make highly parallel multiplexed biosensors and scanning-less spectrometers on a chip. It is important to note that these lasers are still not stand-alone devices because an external gas pressure source is still required to actuate the microfluidic valves, and an external pump laser is required to pump the dye. The gas pressure source can be eliminated by using electrokinetically driven flow, whereas an external pump light will continue to be necessary for our dye laser designs. For portable and inexpensive devices, visible semiconductor lasers or bright light emitting diodes can be used as the pump source.

\section{Acknowledgments}

This research was supported by the DARPA center for optofluidic integration and the Boeing Company under the SRDMA program. 